\title{
Age-Related Change of Pulsatile Gonadotropin Secretion in Turner Syndrome
}

\author{
AKIO HOSODA, KENJI FUJIEDA, NOBUO MATSUURA, AKIHIRO OKUNO, AND KENJI YURI \\ Department of Pediatrics, Hokkaido University, School of Medicine, Sapporo, Japan
}

\begin{abstract}
In an attempt to understand the dynamic change of the gonadotropin-releasing hormone-pituitary axis during the transitional stage from prepuberty to puberty, we investigated gonadotropin secretory patterns using a highly sensitive assay system and frequent blood sampling technique in children with Turner syndrome aged 5-17 y. Blood samples were collected every 20 min for 24 $\mathrm{h}$ in 16 cases, or every $30 \mathrm{~min}$ for $9 \mathrm{~h}$ (daytime $5 \mathrm{~h}$, nighttime $4 \mathrm{~h}$ ) in nine cases. Serum LH and FSH were measured by time-resolved fluoroimmunoassay. A 24-h profile of LH and FSH was analyzed by a computerized pulse detection program (PC-PULSAR). As early as 5 to $6 \mathrm{y}$ of age, mean daytime $\mathrm{LH}$ concentration was significantly higher than nighttime concentration and pulsatile LH secretion existed throughout the day and night. At about 9 to $11 \mathrm{y}$ of age, corresponding to the early stage of puberty, a dramatic increase in $\mathrm{LH}$ concentration and amplitude was observed, and both concentration and pulse amplitude were much higher during the night than during the day. However, these day-night differences became less clear at ages corresponding to late pubertal stages. Pulse frequency of $\mathrm{LH}$ secretion remained almost constant throughout the day and night at all ages investigated. As for FSH concentration, a trend similar to that of $\mathrm{LH}$ was observed, although day-night differences and age-related changes were less remarkable. Furthermore, pulsatile FSH secretion was detected in only a small number of the cases. These findings suggest that in Turner syndrome the hypothalamic gonadotropin-releasing hormone oscillator is functioning actively with constant frequency before the onset of puberty. Although we know that the day-night rhythm of gonadotropin secretion exists long before the onset of puberty, what controls the day-night rhythm of gonadotropin in the younger girls and the factors responsible for the dramatic increase of gonadotropin around the age of early puberty remain to be determined. (Pediatr Res 29: 196-200, 1991)
\end{abstract}

\section{Abbreviations}

Gn-RH, gonadotropin-releasing hormone

The mechanism for the onset of puberty is not yet fully understood, although maturation of the hypothalamic-pituitarygonadal axis plays a major role in initiating the pubertal process. To appreciate the complex mechanism controlling the onset of puberty, it is of great importance to analyze functional changes of the hypothalamus throughout maturational stages of puberty.

Received April 24, 1990; accepted September 25, 1990.

Correspondence and reprint requests: Akio Hosoda, M.D., Department of Pediatrics, School of Medicine, Hokkaido University, North-15 West-7 Kitaku Sapporo, Japan 060.

Supported in part by a Grant for Pediatric Research (63-4) from the Ministry of Health and Welfare, Japan.
It is a well-recognized concept that sleep-enhanced, periodic, pulsatile discharge of $\mathrm{LH}$ is initiated in early puberty $(1,2)$; however, there is no general agreement as to the secretory pattern of gonadotropins in the prepubertal stage before appearance of any appreciable development of secondary sexual characteristics.

In Turner syndrome, where ovarian function is genetically impaired, intensified gonadotropin secretion at the age that corresponds to puberty is also initiated in a similar but more pronounced manner than in normal puberty (3-5). These findings suggest that Turner syndrome represents a typical model of nature to investigate maturation of the Gn-RH-pituitary component of the control system that governs gonadal function under reduced or absent influence of gonadal steroid hormone.

Recently, a very sensitive gonadotropin assay system based on time-resolved fluoroimmunoassay was developed for practical use (6). This assay system is about 100 times more sensitive than previous assay systems. This makes it possible to investigate more definitively the pattern of gonadotropin secretion at low concentration. Accordingly, we used this assay system to investigate age-related changes in the gonadotropin secretory pattern of girls with Turner syndrome. Our special interest was whether or not day-night rhythm and pulsatile gonadotropin secretion exist in prepubertal children.

\section{MATERIALS AND METHODS}

Subjects. Twenty-three girls with Turner syndrome, aged from 5 y 6 mo to 17 y $11 \mathrm{mo}$, were recruited for our study. Diagnosis was confirmed by chromosome analysis of peripheral mononuclear cells. Their karyotypes and clinical characteristics are shown in Table 1. Cases 2 and 8 were studied twice at different ages. None of them showed any spontaneous development of secondary sexual characteristics at the time of our study. Replacement therapy of sex steroid hormones, performed in cases 22 and 23 for the purpose of developing secondary sexual characteristics after they were 15 y old, was discontinued at least several months before the study. Informed consent was obtained from both patients and parents.

Protocol. The experimental protocol was approved by the Human Subjects Investigation Committee of Hokkaido University Hospital. Blood samples were obtained from 16 cases through an indwelling venous catheter every $20 \mathrm{~min}$ for $24 \mathrm{~h}$ using Cormed's pump. From the other nine cases, blood samples were drawn every 30 min manually for $9 \mathrm{~h}$ during daytime hours from 1000 to $1500 \mathrm{~h}$ and during nighttime hours from 2100 to 0100 h. The patients' activities were not restricted. Blood samples were collected into the tubes containing EDTA, and the plasma was separated and frozen until assay. Plasma LH and FSH measurements were performed with a time-resolved fluoroimmunoassay kit (Delfia LH and FSH, Pharmacia, Uppsala, Sweden). Calibrated standards were used for LH against WHO-lst IRP68/40 and FSH against WHO-2nd IRP78/549.

Pulse analysis. Pulse analysis was performed using a computerized program, PC-PULSAR, developed by Merriam and Wachter (7). Detection of pulse peaks of secretion as well as 
Table 1. Subjects studied

\begin{tabular}{|c|c|c|c|c|}
\hline $\begin{array}{c}\text { Case } \\
\text { no. }\end{array}$ & Age & Bone age & $\begin{array}{l}\text { Sampling } \\
\text { condition* }\end{array}$ & Karyotype \\
\hline 1 & 5 y $6 \mathrm{mo}$ & & $\mathrm{A}$ & $45 X / 46 X X$ \\
\hline $2-a$ & 6 y $10 \mathrm{mo}$ & 6 y $6 \mathrm{mo}$ & A & $45 \mathrm{X} / 46 \mathrm{XX}$ \\
\hline 3 & 8 y $7 \mathrm{mo}$ & 6 y $10 \mathrm{mo}$ & B & $46 \mathrm{Xi}(\mathrm{Xq})$ \\
\hline 4 & 8 y $8 \mathrm{mo}$ & 8 y 0 mo & A & $45 \mathrm{X}$ \\
\hline 5 & 9 y $5 \mathrm{mo}$ & 7 y $6 \mathrm{mo}$ & A & $46 \mathrm{Xi}(\mathrm{Xq})$ \\
\hline $8-a$ & 11 y $0 \mathrm{mo}$ & & A & $45 \mathrm{X}$ \\
\hline 9 & 11 y $0 \mathrm{mo}$ & 11 y $0 \mathrm{mo}$ & $\mathrm{B}$ & $46 \mathrm{Xi}(\mathrm{Xq})$ \\
\hline 10 & 11 y $8 \mathrm{mo}$ & 11 y $6 \mathrm{mo}$ & B & $46 \mathrm{X} / 45 \mathrm{X}+\mathrm{mar}$ \\
\hline 11 & 11 y $8 \mathrm{mo}$ & 11 y $0 \mathrm{mo}$ & B & $45 \mathrm{X}$ \\
\hline 12 & 11 y $11 \mathrm{mo}$ & 11 y $0 \mathrm{mo}$ & $\mathrm{B}$ & $45 \mathrm{X}$ \\
\hline 13 & 11 y $11 \mathrm{mo}$ & 10 y $0 \mathrm{mo}$ & $\mathrm{B}$ & $45 X$ \\
\hline $8-b$ & 13 y $7 \mathrm{mo}$ & & $\mathrm{B}$ & \\
\hline 18 & 13 y $8 \mathrm{mo}$ & & A & $45 \mathrm{X}$ \\
\hline 19 & 14 y $7 \mathrm{mo}$ & 11 y $0 \mathrm{mo}$ & A & $45 X / 46 X Y q-$ \\
\hline 20 & 14 y $7 \mathrm{mo}$ & & $\mathrm{A}$ & $45 X$ \\
\hline 21 & 14 y $8 \mathrm{mo}$ & 13 y $0 \mathrm{mo}$ & B & $45 \mathrm{X} / 46 \mathrm{X}+\mathrm{mar}$ \\
\hline 22 & 16 y $6 \mathrm{mo}$ & 15 y $0 \mathrm{mo}$ & $\mathrm{B}$ & $45 \mathrm{X} / 46 \mathrm{X}, \mathrm{r}(\mathrm{X})$ \\
\hline 23 & 17 y $11 \mathrm{mo}$ & 13 y $10 \mathrm{mo}$ & $\mathrm{A}$ & $45 \mathrm{X} / 46 \mathrm{Xi}(\mathrm{Xq})$ \\
\hline
\end{tabular}

* A, 9 h every $30 \mathrm{~min}$; B, $24 \mathrm{~h}$ every $20 \mathrm{~min}$.

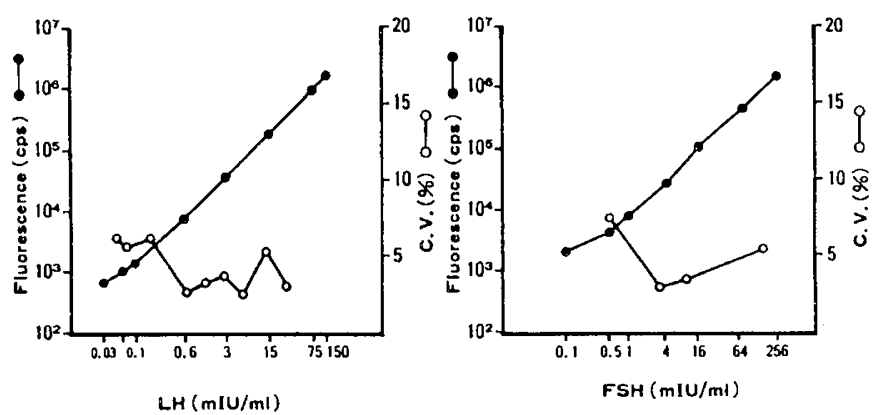

Fig. 1. Standard curves and assay coefficients of variation of timeresolved fluoroimmunoassay for $\mathrm{LH}$ and $\mathrm{FSH}$.

calculation of mean pulse amplitude was performed only for the cases with 20 -min sampling schedules for $24 \mathrm{~h}$. Default values of parameters for calculation in PC-PULSAR were not modified other than those for least detectable concentration. Assay SD approximated by a linear function of concentration constructed from the values of SD over the measured ranges of concentration to be analyzed were used as a scale factor for the detection of peaks in the program.

Figure 1 shows the standard curves and assay coefficients of variation at various concentrations of $\mathrm{LH}$ and FSH. Intraassay coefficient of variation was $2-7 \%$ for $\mathrm{LH}$ and $3-8 \%$ for $\mathrm{FSH}$ over the wide range of concentrations. Assay sensitivity, determined as twice the SD at zero concentration, was $0.03 \mathrm{IU} / \mathrm{L}$ for $\mathrm{LH}$ and $0.1 \mathrm{IU} / \mathrm{L}$ for FSH.

Mean concentration during daytime (0800 to $2000 \mathrm{~h})$ and nighttime (2000 to $0800 \mathrm{~h}$ ) was calculated in every case.

Statistical analysis. Wilcoxon's rank-sum test was used to verify the difference between day and night concentrations.

\section{RESULTS}

Representative examples of actual gonadotropin secretory patterns are shown in Figure $2 A-D$. Individual results of the day and night concentrations and pulse analysis of gonadotropins are summarized in Tables 2 and 3. All the girls with Turner
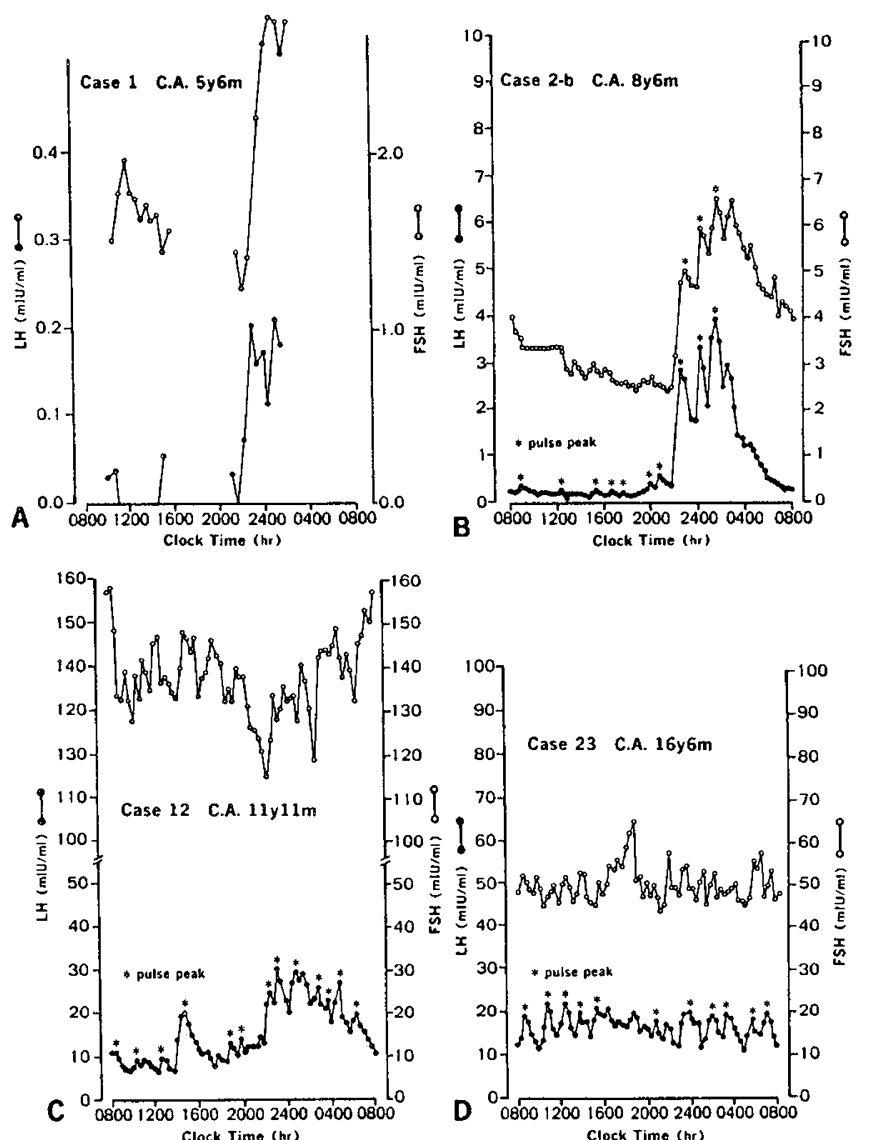

Fig. 2. Representative examples of gonadotropin secretory pattern. Vertical scales of concentration are not uniform. At the age of early prepuberty, day-night rhythm of gonadotropins, especially $\mathrm{LH}$, is already seen, although their absolute value is low $(A, B)$. After the age of early puberty, gonadotropin concentrations become much higher $(C)$; however, day-night rhythm becomes vague by the age of late puberty $(D)$. Pulse peaks of LH are seen during both day and night and through all ages. 
Table 2. Mean concentration, number of pulse peaks, and mean pulse amplitude of $L H$

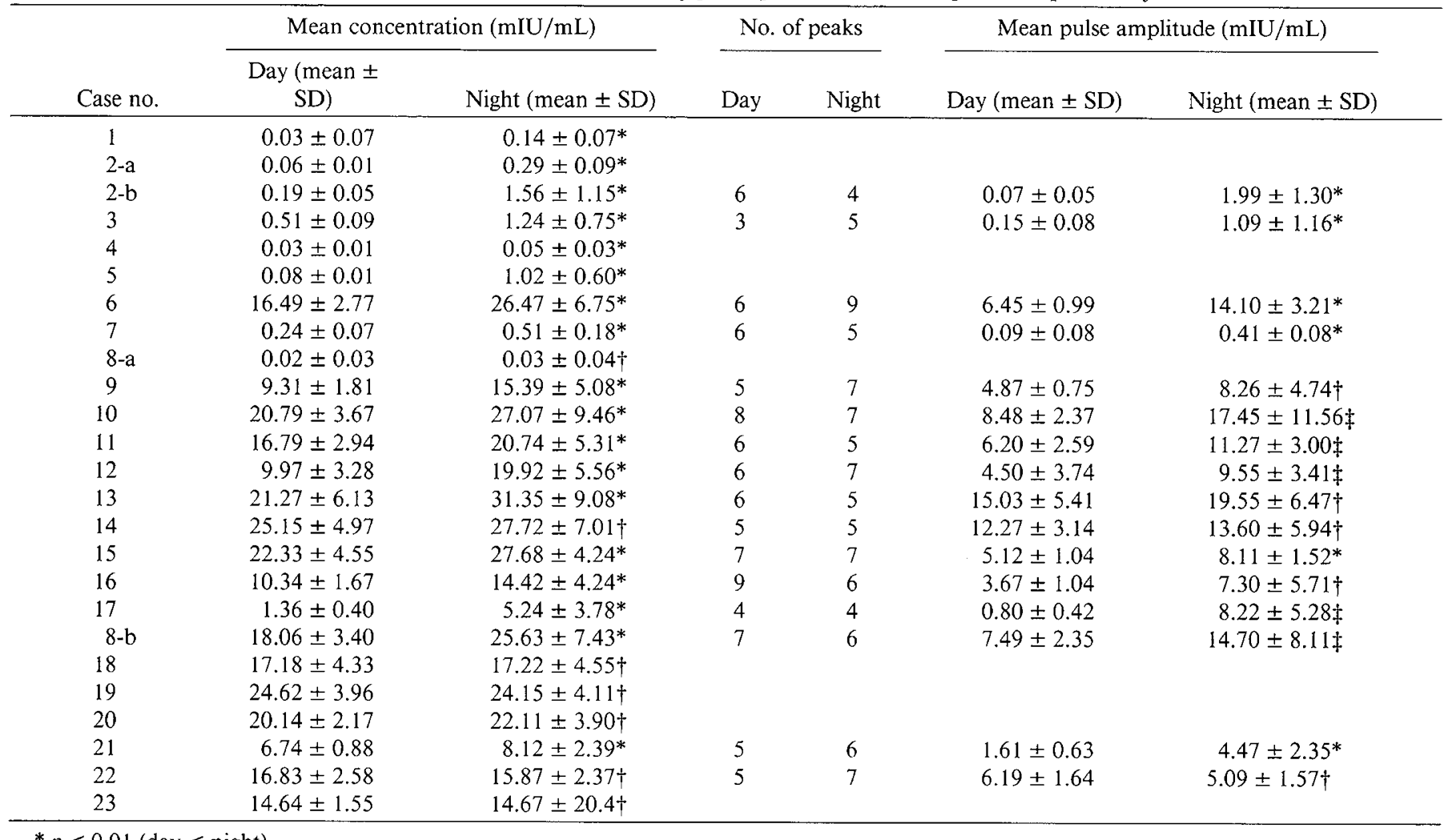

$* p<0.01$ (day $<$ night).

$\dagger \mathrm{NS}$.

$\ddagger p<0.05$ (day $<$ night).

Table 3. Mean concentration, number of pulse peaks, and mean pulse amplitude of FSH

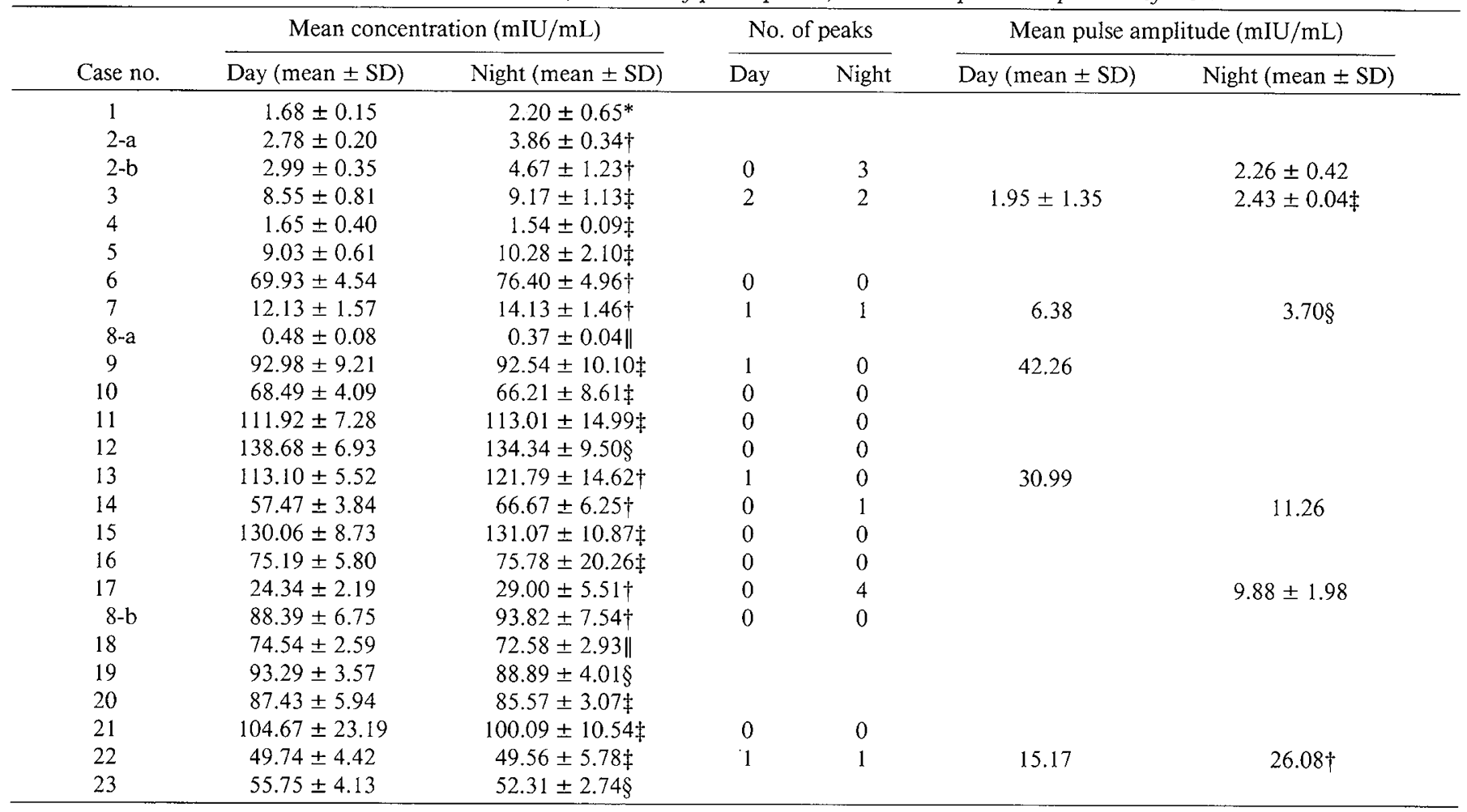

$* p<0.05$ (day $<$ night).

$\dagger p<0.01$ (day $<$ night).

$\ddagger$ NS.

$\S p<0.01$ (day $>$ night).

$\| p<0.05$ (day $>$ night). 

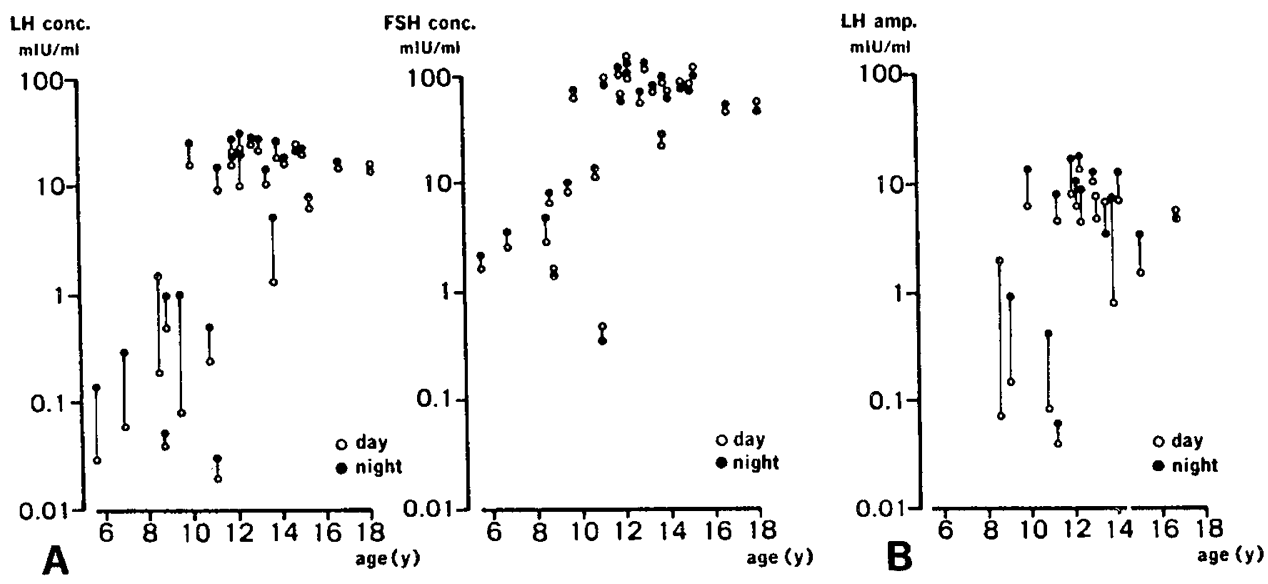

Fig. 3. A, individual day and night mean concentrations of LH and FSH. Vertical axis drawn in logarithmic scale. Concentration of LH increases dramatically around the ages of 9 to 11 y. $B$, individual day and night mean amplitudes of LH pulse. The amplitude increases coinciding with the concentration.

syndrome from prepuberty to puberty showed episodic LH secretion. Also, distinct day-night rhythm of $\mathrm{LH}$ was seen in most cases of prepubertal age.

Nighttime concentration of LH was significantly higher than daytime concentration in younger aged cases whose levels of $\mathrm{LH}$ concentration were relatively low. Both day and night, mean concentration increased dramatically around the ages of 9 to 11 $y$. The day-night concentration difference became less clear in older age cases (Fig. 3A). Pulse peaks of LH were detected throughout the day and night in every case investigated. Furthermore, the profile of LH in cases 1 and 2 suggested existence of pulsatility, although their 30-min sampling interval was too long to analyze by PC-PULSAR (Fig. $2 A$ ). Mean pulse amplitude of LH correlated with corresponding LH concentration, and amplitude also increased around the ages of 9 to $11 \mathrm{y}$, following the same trend as LH concentration (Fig. $3 B$ ). Pulse frequency of $\mathrm{LH}$ was $10-15$ peaks $/ 24 \mathrm{~h}$ (mean $11.8 \pm 2.2 / 24 \mathrm{~h}$ ), and this frequency was independent of age or the concentration of $\mathrm{LH}$. Distribution of pulse peaks during every $4 \mathrm{~h}$ stayed almost constant throughout the day (from $1.7 \pm 0.8$ during h 12-16 to $2.1 \pm 1.0$ during $h$ 16-20). Case 17 showed relatively low concentration and amplitude of $\mathrm{LH}$ for her chronologic age, although her bone age was somewhat younger.

FSH showed a trend similar to that of LH (Fig. $3 A$ ). However, the increase of FSH concentration around the age of early puberty was less dramatic. Day-night difference was also less prominent. FSH concentration was relatively high compared to $\mathrm{LH}$ concentration in the younger group of cases. Only small numbers of FSH pulse peaks, almost all of which coincided with $\mathrm{LH}$ pulse peaks, were detected in several cases.

\section{DISCUSSION}

By using frequent blood sampling, a highly sensitive gonadotropin assay, and a computerized pulse detection algorithm, we demonstrated that significant day-night rhythm of LH concentration exists at the prepubertal stage as early as $5 \mathrm{y}$ of age in girls with Turner syndrome. Furthermore, we demonstrated that, at the age of $8 \mathrm{y}$ or more, LH secretion was pulsatile in nature. Pulse frequency did not change throughout the transition phase from prepuberty to puberty. In addition, marked increment of LH concentration around the age of early puberty coincided with a marked increase of pulse amplitude. At the stage of late puberty, day-night difference of $\mathrm{LH}$ concentration became less prominent.

Similar findings in principle were reported by Ross et al. (8). However, there are minor differences between their findings and ours. We observed more marked day-night rhythmicity in prepubertal stages. Furthermore, no apparent difference of pulse frequency of LH was observed during the stages of pubertal development. Ross claimed that the combined pulse frequency from both LH and FSH did not change, although LH frequency increased and FSH frequency decreased with advancing age. These differences might be due to several factors such as difference in assay systems or methods of pulse analysis. It is most probable that the conventional RIA methods used by Ross et al. (8) were not sensitive enough to evaluate lower LH concentrations. This might lead to underestimation of day-night difference in $\mathrm{LH}$ concentration and LH pulse frequency in the prepubertal stage.

Assuming that the pattern of $\mathrm{LH}$ release from the pituitary gland closely reflects the pattern of $\mathrm{Gn}-\mathrm{RH}$ release from the hypothalamus (9), our results suggest that the hypothalamic oscillator mechanism is functioning far before the onset of puberty, at least in Turner syndrome.

Whether day-night rhythm and pulsatility of gonadotropin secretion exists in normal prepubertal children is still not settled. However, the more sensitive the assay system used, the more day-night rhythm and pulsatility found. For instance, Jackachi et al. (10) found day-night rhythm and pulsatile secretion of $\mathrm{LH}$ in half of the prepubertal children with bone age from 3 to $9.5 \mathrm{y}$ by using a conventional RIA system but improving its sensitivity several-fold. Furthermore, Wennik et al. (11), using an immunoradiometric assay with a sensitivity of 0.1 IU/L of 1st IRP68/ 40 , detected nocturnal pulsatility of $\mathrm{LH}$ in three normal prepubertal boys, whereas LH levels during the daytime remained below assay sensitivity. It is probable that pulsatility might have been detected in the daytime if the assay were sensitive enough.

From a viewpoint of hormonal environment, Turner syndrome has reduced feedback effect of gonadal steroids compared to that in normal children. It might be interesting to know how sex steroid hormones modulate episodic gonadotropin secretory pattern. Indeed, Mauras et al. (12) found that a low dose of estrogen administered to prepubertal girls with Turner syndrome reduced gonadotropin concentration and pulse amplitude, but did not modulate pulse frequency. If additional studies confirm that day-night rhythm and pulsatile secretion of gonadotropins exist in both normal prepubertal children and prepubertal girls with Turner syndrome and a similar pronounced increase in secretion is seen at the onset of puberty, changing level of threshold to the gonadal steroid hormones in the hypothalamus and pituitary gland might not play a major role in the onset of puberty.

The hypothalamic-pituitary component of the control system 
that governs gonadal function attains a high degree of organization already during the fetal period. Also, it was recently reported that chemical stimulation of the hypothalamus by N-methyl-Dasparic acid can easily result in precocious puberty through activation of the Gn-RH-pituitary system in rat and rhesus monkey $(13,14)$. In light of these findings, our results may indicate that the difference between prepubertal and pubertal states is not qualitative but rather quantitative in nature.

Acknowledgments. The authors thank Mrs. Fujishima and Miss Tokuchi for their technical assistance. The authors are also grateful to Professor Matsumoto for invaluable advice in reviewing this manuscript.

\section{REFERENCES}

1. Boyar RM, Finkelstein J, Roffbang H, Kapen S, Weitzman E, Hellman L 1972 Synchronization of augmented luteinizing hormone secretion with sleep during puberty. N Engl J Med 287:582-586

2. Boyar RM, Rosenfeld R, Kapen S, Finkelstein J, Roffwark H, Weitzman E, Hellman L 1974 Human puberty-simultaneous augmented secretion of luteinizing hormone and testosterone during sleep. J Clin Invest 54:609-618

3. Boyar RM, Ramsay J, Chipman J, Febre LM, Madden J, Marks JF 1978 Luteinizing hormone and follicle-stimulating hormone secretory dynamic in Turner's syndrome. J Clin Endocrinol Metab 47:1078-1083

4. Conte FA, Grumbach MM, Kaplan SL 1975 A diphasic pattern of gonadotro- pin secretion in patients with the syndrome of gonadal dysgenesis. $\mathrm{J}$ Clin Endocrinol Metab 40:670-674

5. Urban MD, Migion CL, Lee PA 1981 Pubertal secretory pattern of gonadotropins in hypergonadotropic patients (Turner syndrome). Hormone Res 14:209-214

6. Lovgren T, Hemmilia I, Petterson K, Jarkko U, Bertoft E 1984 Determinations of hormones by time-resolved fluoroimmunoassay. Talanta 31(10B):909916

7. Merriam GR, Wachter KW 1982 Algorithms for the study of episodic hormone secretion. Am J Physiol 243:310-318

8. Ross JL, Loriaux DL, Cutler Jr GB 1983 Development change in neuroendocrine regulation of gonadotropin secretion in gonadal dysgenesis. $\mathbf{J}$ Clin Endocrinol Metab 57:288-293

9. Clarke IJ, Cummons JT 1982 The temporal relationship between gonadotropin releasing hormone (GnRH) and luteinizing hormone (LH) secretion in ovariectomized ewes. Endocrinology 111:1737-1739

10. Jackachi RI, Kelch RP, Sauder SE, Loid LS, Hopwood NJ, Marshall JC 1982 Pulsatile secretion of luteinizing hormone in children. J Clin Endocrinol Metab 55:453-457

11. Wennik JMB, Delemarre-van de Wall HA, van Kessel H, Mulder GH, Foster JP, Shoemaker J 1988 Luteinizing hormone secretion in boys at the onse of puberty measured using a highly sensitive immunoradiometric assay. J Clin Endocrinol Metab 67:924-928

12. Mauras N, Rogal AD, Veldhuis JD 1989 Specific time-dependent actions of low-dose ethinyl estradiol administration on the episodic release of growth hormone, follicle-stimulating hormone and luteinizing hormone in prepubertal girls with Turner's syndrome. J Clin Endocrinol Metab 69:1053-1058

13. Urabanski HF, Ojieda SR 1987 Activation of luteinizing hormone release advances the onset of female puberty. Neuroendocrinology 46:273-276

14. Plant TM, Gay VL, Marshall GR, Arslan M 1989 Puberty in monkeys is triggered by chemical stimulation of the hypothalamus. Proc Natl Acad Sci USA $86: 2506-2510$ 